\title{
¿Qué tipo de innovación desarrollar?
}

En los últimos años, se ha observado un esfuerzo creciente por promover la innovación en las empresas con el objeto de aumentar su competitividad. Sin embargo, es necesario precisar el tipo de innovación que éstas deberían desarrollar, porque sus efectos en el desempeño empresarial difieren dependiendo del sector productivo en que ésta se desarrolle. En este contexto, el objetivo de este artículo es presentar distintos tipos de innovación y su relevancia en distintos sectores de la economía chilena.

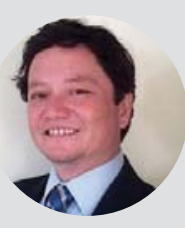

Cristian Geldes

PROFESOR DE MANAGEMENT DEPARTAMENTO DE GESTION Y NEGOCIOS, FEN-UAH

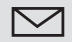

cgeldes@uahurtado.cl

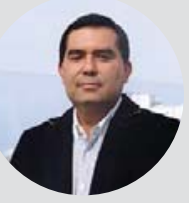

Jorge Heredia PROFESOR DE ESTRATEGIA, UNIVERSIDAD DEL PACÍFICO, PERÚ

$\triangle$

ja.herediap@up.edu.pe

$C\left(C_{1}\right)$

El impacto de distintos tipos de innovacion depende del sector productivo en que opera la empresa ")

\section{Introducción}

La innovación es clave para promover el crecimiento económico y la competitividad. Por esta razón, se han impulsado una serie de políticas y programas para impulsar su fomento tanto en el ámbito público como el privado; siendo un tema recurrente en discursos y definiciones de políticas públicas y estrategias empresariales. Aunque pocos objetan la necesidad de innovar, es necesario avanzar en la distinción de los tipos de innovaciones que pueden realizar las empresas y el eventual impacto en su desempeño. En este contexto, este artículo busca contribuir a la discusión respecto del tipo de innovación que deben realizar las empresas y a la necesidad de generar programas de fomento específicos para los distintos tipos de innovación, de manera que estos consideren diferencias sectoriales. A continuación se presenta una revisión de las principales clasificaciones de los tipos de innovación, un análisis de los distintos efectos en el caso chileno $y$, por último, se presentan reflexiones y sugerencias para gestores de políticas públicas y managers.

\section{Tipos de innovación}

La innovación ha sido definida y analizada desde diferentes perspectivas. Estas incluyen enfoques empresariales y enfoques sistémicos como son los clusters, distritos industriales, sistemas regionales de innovación, especialización inteligente entre otros (Crossan y Apaydin, 2010; McCann y Ortega-Argilés, 2015). En el caso específico de las innovaciones a nivel empresarial, se han desarrollado una serie de clasificaciones que se presentan en la Tabla 1. Este proceso ha evolucionado desde tipologías más bien dicotómicas como son las innovaciones administrativas y técnicas o incrementales y radicales, a clasificaciones más complejas (Rowley et al., 2011). 


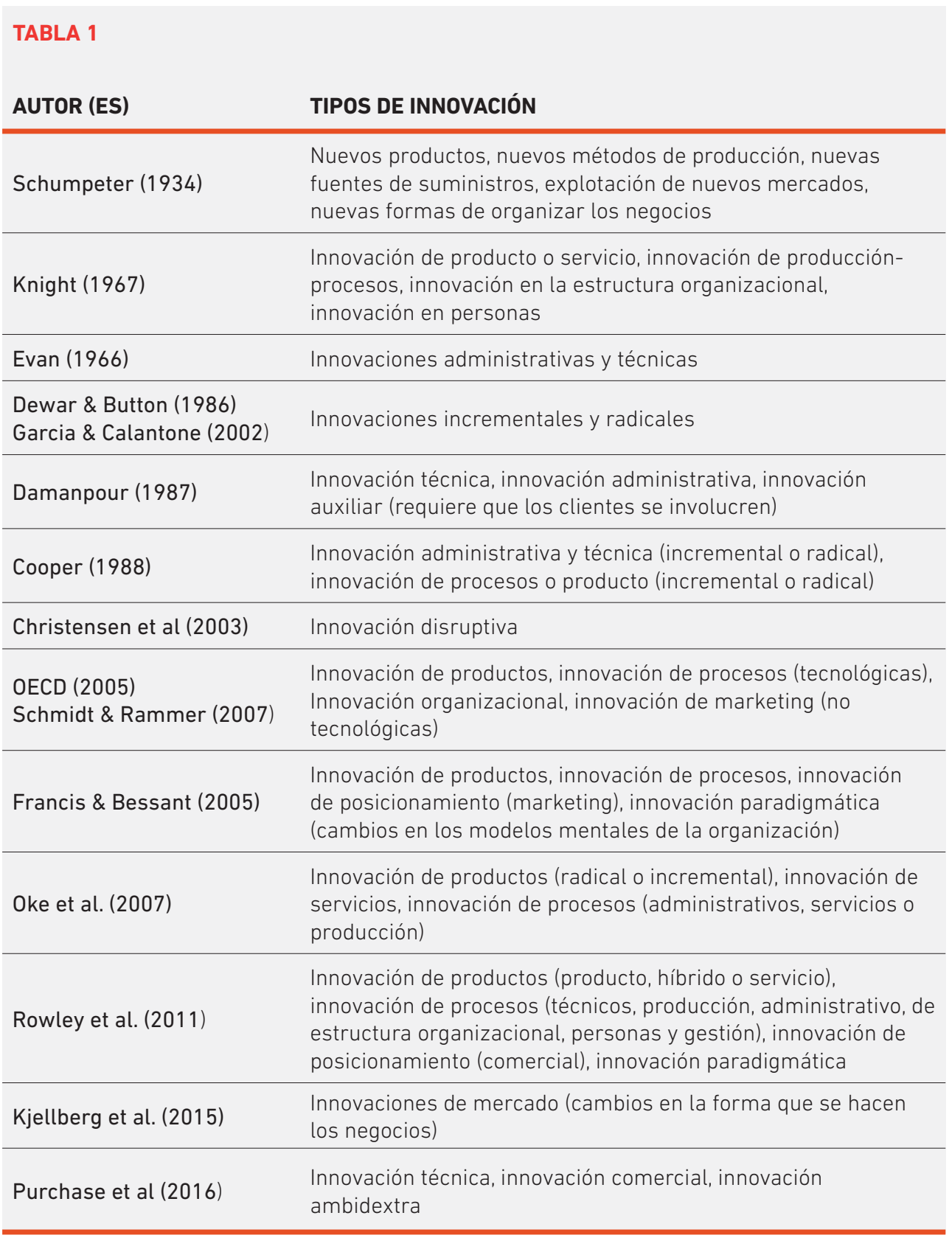

\section{Tipos de innovación identificados en la literatura}

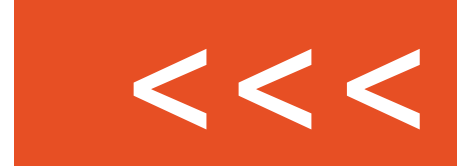

Los múltiples tipos de innovación muestran que el llamado a las empresas a innovar no es preciso. Cada tipo de innovación requiere de un modelo específico de gestión, en el que confluyen distintos recursos, capacidades y redes de colaboración (Tidd y Bessant, 2014). Además, las distintas innovaciones tienen distintos determinantes y factores que favorecen su desarrollo, como el tamaño de la empresa, si exporta, si realiza investigación y desarrollo, capacidad de acceso a fuentes de información y financiamiento, entre otros (Schmidt y Rammer, 2007; Geldes y Felzensztein, 2013). Del mismo modo, se suma el hecho que las innovaciones varían en función del territorio y por cada sector económico (Hall y Rosenberg, 2010). En conjunto, estos elementos plantean la necesidad de conocer en más detalle los efectos de las distintas innovaciones en el desempeño de la empresa.

\section{Innovaciones tecnológicas y no tecnológicas}

Frente a los diversos tipos de innovación, es conveniente considerar la propuesta del Manual de Oslo (OECD, 2005) que se usa como base para el diseño de las encuestas nacionales de innovación de los países miembros de la OECD, incluido Chile. En este manual se distinguen cuatro tipos de innovaciones que se agrupan en: 
innovaciones tecnológicas (innovaciones de productos e innovaciones de procesos) e innovaciones no tecnológicas (innovaciones en marketing e innovaciones organizacionales).

Para analizar los efectos de los distintos tipos de innovación en la empresa, se considera el estudio "Technological and non-technological innovation, performance and propensity to innovate across industries: The case of an emerging economy" (Geldes et al., 2017). Este estudio analiza los efectos de los distintos tipos de innovaciones en el desempeño innovador y en la propensión a innovar en empresas de los sectores agrícola, manufacturero y servicios. Los datos del estudio corresponden a 2.549 empresas que respondieron la VI Encuesta Nacional de Innovación (2015).

La Tabla 2 presenta los resultados del análisis del efecto de las innovaciones en el desempeño innovador, medido como la relación entre ventas de productos innovadores y los productos totales de la empresa. Se observa que la innovación en productos juega un rol relevante en las industrias de manufactura y de servicios, y en las tres industrias de forma agregada, lo cual es esperable. Del mismo modo, se aprecia que la innovación organizacional es relevante para el caso de la industria manufacturera. Esto sugiere que los cambios que se realicen en nuevas prácticas y métodos de administración, así como en las relaciones con otros agentes, han tenido un impacto positivo en el desempeño innovador.

\section{Relación entre desempeño innovador $y$ tipos de} innovaciones

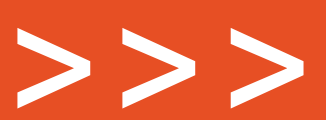

FUENTE: ADAPTADO DE GELDES ET AL, 2017.
TABLA 2

\section{VARIABLES AGRICULTURA MANUFACTURAS SERVICIOS TRES INDUSTRIAS}

\begin{tabular}{lccc}
$\begin{array}{l}\text { Innovación en } \\
\text { productos }\end{array}$ & $*$ & $*$ & $*$ \\
\hline $\begin{array}{l}\text { Innovación en } \\
\text { procesos }\end{array}$ & $*$ & & \\
\hline $\begin{array}{l}\text { Innovación } \\
\text { organizacional }\end{array}$ & & & \\
\hline $\begin{array}{l}\text { Innovación en } \\
\text { marketing }\end{array}$ & & & $*$ \\
\hline Ventas & $*$ & $*$ & $*$ \\
\hline Exportaciones & $*$ & & \\
\hline I+D & & & \\
\hline
\end{tabular}

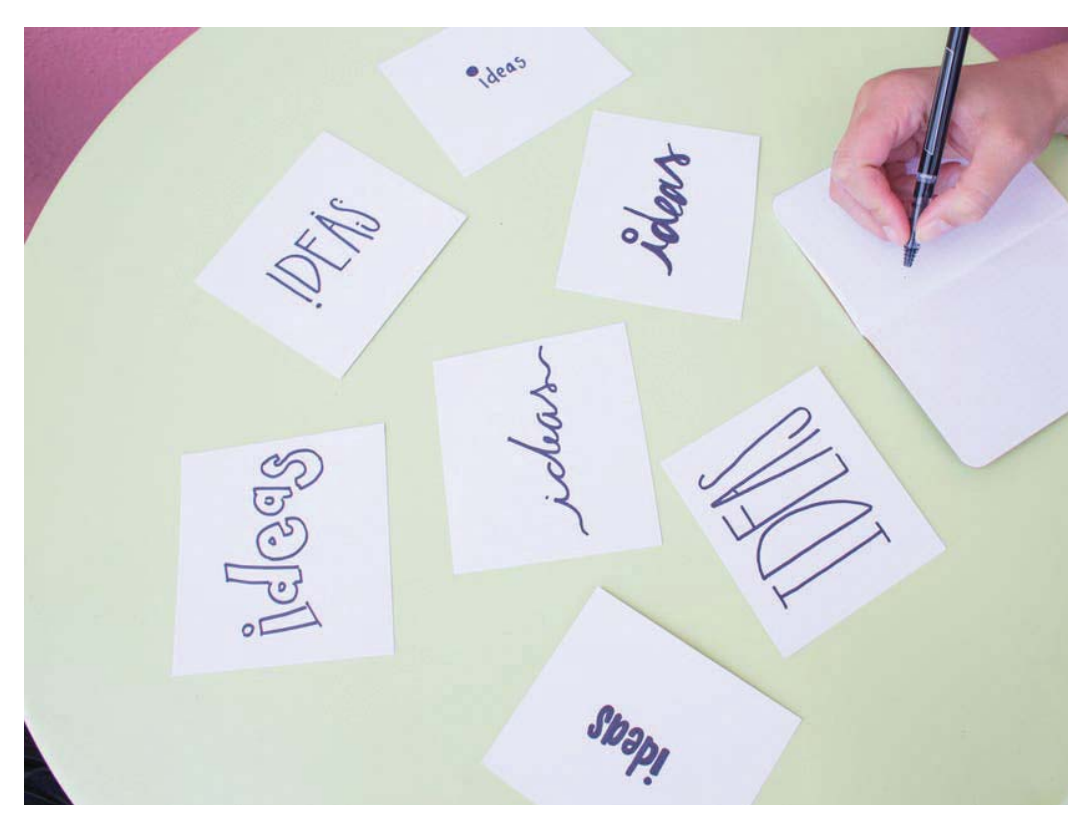

La Tabla 3 presenta los resultados de estimaciones del efecto de las innovaciones en la propensión a innovar, es decir, en el desarrollo futuro de innovaciones. Se observa que las innovaciones juegan un rol diferente según el tipo de propensión a innovar. Por ejemplo, en el caso de la propensión a innovar en procesos ("Proc"), se ve que la innovación organizacional es relevante para el conjunto de las tres industrias, y en los casos específicos de las industrias de manufacturas y servicios. Esto sugiere que las nuevas prácticas y métodos en administración son relevantes para la innovación en procesos. A su vez se establece que las empresas de las industrias de manufacturas y servicios que realizan innovaciones en marketing, en el futuro lo volverán a hacer. Por último, se observa que la innovación de procesos es clave para realizar innovaciones organizaciones en la agricultura y en el conjunto de las tres industrias. 
TABLA 3

\begin{tabular}{l|c|c|c|c|c|c|c|c|c|c|c|c}
\hline & \multicolumn{1}{c}{ PROPENSIÓN A INNOVAR/SECTOR } \\
\cline { 2 - 12 } $\begin{array}{l}\text { TIPOS DE } \\
\text { INNOVACIONES }\end{array}$ & \multicolumn{1}{c}{ AGRICULTURA } & \multicolumn{1}{c}{ MANUFACTURAS } & \multicolumn{5}{c}{ SERVICIOS } \\
\cline { 2 - 13 } & Pdto Proc & Org & Mkt & Pdto & Proc & Org & Mkt & Pdto & Proc & Org & Mkt \\
\hline Productos & & & & & $*$ & & & & $*$ & $*$ & $*$ & \\
\hline Procesos & & & $*$ & & & & & & & $*$ & & \\
\hline Organizacional & & & & $*$ & $*$ & $*$ & $*$ & & & $*$ & $*$ & \\
\hline Marketing & & & & & & & & $*$ & & & & $*$ \\
\hline
\end{tabular}

"Pdto": Propensión a innovar en productos; "Proc": Propensión a innovar en procesos; "Org": Propensión a innovaciones organizacionales; "Mkt": Propensión a innovar en marketing. Fuente: Adaptado de Geldes et al (2017).

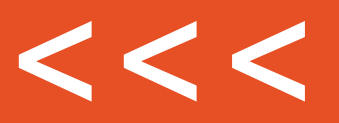

\section{Reflexiones finales}

Los resultados anteriores muestran que el efecto de los distintos tipos de innovación varía según el sector económico, tanto en el desempeño innovador como en la propensión a innovar. En este contexto, los llamados a "innovar" requieren enfatizar estas diferencias tanto para el diseño de políticas y programas públicos de fomento, como en la elaboración de estrategias empresariales. Específicamente, las políticas para fomentar la innovación debieran considerar diferencias sectoriales. Un ejemplo de esto es lo que hoy se realiza en el Fundación para la Innova- ción Agraria (FIA), que ha desarrollado distintos instrumentos sectoriales tanto para el desarrollo de productos como para innovar en marketing. Sin embargo, es necesario profundizar en esta distinción en otros programas de fomento.

Por otra parte, para los managers o administradores los resultados de la propensión a innovar muestran una cierta secuencia que debieran seguir las empresas para mejorar su desempeño innovador. Es decir, estos sugieren que innovaciones realizar para innovar en productos, lo que final- mente es el resultado más atractivo desde el punto de vista de la rentabilidad de la empresa, y el cual no se puede generalizar para todos los sectores económicos.

Finalmente, surgen una serie de preguntas: ¿En qué medida los programas de fomento de innovación consideran las diferencias sectoriales y territoriales? ¿Qué innovaciones se consideran relevantes en las evaluaciones de los programas competitivos que fomentan la innovación? ¿Las empresas han articulado una ruta para implementar distintos tipos de innovaciones?.

\section{Referencias}

Cooper, J. R. (1998). A multidimensional approach to the adoption of innovation. Management Decision, Vol. 36, No. 8, 493-502

Crossan, M.M. y Apaydin, M. (2010). A multi-dimensional framework of organizational innovation: A systematic review of the literature. Journal of Management Studies, Vol.47 No.6, 1154-1191.

Damanpour, F. (1987). The adoption of technological, administrative and ancillary innovations: impact of organizational factors. Journal of Management, Vol. 13, No. 4, 675-688.

Deward, R. D. y Button, J. E. (1986). The adoption of radical and incremental innovations: an empirical analysis. Management Science, Vol. 32, No. 11, 1422-1433

Evan, W. M. (1966). Organizational lag. Human Organizations, Vol 25, No. 1. 51-53.
Francis, D. y Bessant, J. (2005). Targeting innovation and implications for capability Development. Technovation, Vol. 25, No. 3, 171-183.

Garcia, R. y Calantone, R. (2002). A critical look at technological innovation typology and innovativeness terminology: a literature review. Journal of Product Innovation Management, 19, 110-132.

Geldes, C. y Felzensztein, C. (2013) Marketing innovations in the agribusiness sector. Academia Revista Latinoamericana de Administración, Vol.26. No.1, 108-138.

Geldes, C., Felzensztein, C. y Palacios, J. (2017). Technological and non-technological innovations performance and propensity to innovate across industries. The case of an emerging economy. Industria Marketing Management, 61, 55-66.

Hall, B. y Rosenberg, N. (2010). Introduction to Handbook., Hall, B. and Rosenberg.
N. (Eds.), The Handbook of Economics of Innovation, Vol. 1, Elsevier, NorthHolland Publications, Amsterdam, 3-9.

Kjellberg, H. Azimont, F. y Reid, E. (2015). Market innovation processes: Balancing stability and change, Industrial Marketing Management, 44, 4-12.

Knight, K. E. (1967). A descriptive model of intra-firm innovation process. Journal of Management, Vol. 40, No. 4, 478-496.

McCann, P. y Ortega-Argilés (2015). Smart specialization, regional growth and applications to European Union Cohesion Policy. Regional Studies Vol. 49. Iss. 8, 1291-1302.

OECD, 2005. Oslo Manual: Guidelines for Collecting and Interpreting Innovation Data (3rd Edition). OECD.

Oke, A., Burke, G. y Myers, A. (2007). Innovation types and performance in growing UK SMEs. International Journal of Operations \& Production Management, 27 (7), 735-753.
Purchase, S., Kumn, C. y Olaru, D. (2016) Paths, events and resource use New developments in understanding innovation process. Industrial Marketing Management, 58, 123-136.

Rowley, J., Bargemen, A. y Sam Brook, S. (2011). Towards an innovation-type mapping tool. Management Decision, 49 (1), 73-86.

Schmidt, T. y Rammer, C. (2007) Non-technological and technological innovation: strange bedfellows? Discussion Paper 07-052, ZEW-Centre for European Economic Research Mannheim, Germany, 11 September.

Schumpeter, Joseph. 1934. The theory of economic development: an inquiry into profits, capital, credit, interest and business cycle. Harvard economic studies, 46. Harvard University Press. 255

Tidd, J. y Bessant, J. (2014). Strategic innovation management. Wiley. 417 p. 